\title{
Study and Design of a Magnetic Levitator System
}

\author{
Brian Meneses-Claudio ${ }^{1}$, Avid Roman-Gonzalez ${ }^{3}$ \\ Image Processing Research Laboratory (INTI-Lab) \\ Universidad de Ciencias y \\ Humanidades \\ Lima, Perú
}

\author{
Zeila Torres Santos ${ }^{2}$ \\ Interdisciplinary Research Center Science and Society \\ (CIICS), Universidad de Ciencias y \\ Humanidades \\ Lima, Perú
}

\begin{abstract}
Magnetic levitation is one of the mechanisms that is at the forefront of technology. It is used in its most basic form in educational teaching, where the principles of physics converge that have as their principle electromagnetism and the fields created by existing poles that repel according to a quantity of initial current, giving instructive ideas of how the theoretical formulas work, giving life to a practical visual system. The current use on a large scale are the Maglev trains of Japan or superconductivity, being the realization of the quantum effects visualized at the moment of cooling the sample. The electronic circuit tends to be stable because, when using a high-power current, a Triac is needed to compensate the electrical flow provided by the operational amplifier and, therefore, stabilize with the photodiode when activated with the Led diode. Our purpose is to create a circuit that identifies the values of the electronic components that allow reaching equilibrium, with input and output variables that indicate the position and height of the object to be levitated.
\end{abstract}

Keywords-Magnetic levitator; electromagnet; electronic circuit; differential potential

\section{INTRODUCTION}

The magnetic and gravitational force, will allow us to understand the Levitation phenomenon of great importance and visually enrich.

The Magnetic Levitator [1] teaches us not only how magnetic fields are generated [2] and the repulsion force that the system must have in order to avoid the force of gravity that all bodies are affected. This is the starting point to understand how a body could levitate. For this purpose, will be necessary to create a functional circuit with electronic devices and coils, when the electric current passes, allows us to see the object suspended. An example of utility is the super-fast public transport trains in Japan.

At the beginning of the seventeenth century, researchers such as Volta, W. Gilbert and others-maintained ideas that Electricity and Magnetic were understood separately, until at least the nineteenth century that Oersted showed a connection between the magnetic field and the electric field using a compass and a conductive thread. Then, in 1861 with Maxwell, he joined the concepts with specific formulas that were fundamental to enrich that area, concepts such as magnetic induction, magnetic permeability, magnetic field, automatic inductance and more. The implementation of the formulation to the construction of the levitator came together with the theory of the Electromagnetism and depending on the type of levitator that you want to work come together mathematical equations that help to discover with accuracy the physical quantities of voltages, sensitivities, electronic units and other useful components in the process [3].

Levitate an object is possible, and there are variants within the electromagnetic theory to perform this process. Already by itself only an electromagnet and its ferromagnetic core forms the basis for levitating an object, with an array of polarities of the metallic object. A current flow through the wound coils of the electromagnet generating a magnetic field, being that the ferromagnetic core provides a path of reluctance in which the magnetic field is concentrated which induces an attractive force on the object to be levitated. Now, if we understand the theory of Superconductivity [4], we know that cooling an object at sufficiently low temperatures causes a distance to be separated from its initial base by positioning itself at a height that we can call a gap, until the temperature again decays. There is levitation by repulsion and suspension, in both cases a study of field forces must be performed, because positioning the object at the midpoint that will be the equilibrium-stable point to levitate, will depend a lot on the circuit, the coils and the distance between them, how to calibrate until the objective is achieved. When we speak of coils through them, an electric field flows generating a force field, requiring an adjustment in the flowing current.

The power electronics has a very large study area, the most important being the control of the current, because of this TRIACS are used in addition to resistors for the opposition to the current [5].

Photodiodes are used for the activation and opening of systems by means of the excitation of light being directly proportional, meaning that when the photodiode is excited with the intensity of light, its impedance will decrease increasing the circulation of current [6].

The following research work is structured as follows: In Section II, the methodology for the design of a magnetic levitator circuit will be presented, for which, equations are proposed on the magnetic force for the estimation of electronic components required for magnetic levitator also shows the design of the simulated electronic circuit in the Proteus 8 software. In Section III, the faults that were obtained at the time of the simulation are briefly explained indicating that no current losses were obtained. In Section IV, the discussion of the research work is presented, as well as the problems that were obtained for the design of the magnetic levitator circuit. Finally, in Section V, the conclusions of the research work are presented. 


\section{Methodology}

\section{A. Magnetic Levitator}

Since magnetism is a physical property of response to the applied magnetic field, it is measured through the Lorentz Force, given by the following equation:

$\vec{E}=\frac{\overrightarrow{\mathbb{F}}}{q}$

What produces the magnetic field are the electric currents or electric field $\vec{E}$ that varies with time, being the charge $q$ in Coulomb that moves with a given electric force $\overrightarrow{\mathbb{F}}$ in Newton.

Therefore, if we join these two fields being one dependent on the other, we obtain the electromagnetic force by the following equation.

$\vec{F}=q \vec{E}+q \vec{v} \times \vec{B}$

Where $\vec{B}$ it is the magnetic field vector.

As the equations begin to arise, the system could be linear, non-linear, MISO (multiplexer), SIMO (demultiplexer), SISO (single input and single output), variant, invariant or depending on the inputs and outputs of the system, forming a set of state equations that describe the dynamics of the system, which in a general way describe the movement and functionalities in conjunction with the variables that make up the system [7]:

$\dot{x}(t)=f(x, u, t)$

$\ddot{x}(t)=h(x, u, t)$

These systems equation is depending on the position $(x)$, current-voltage $(u)$ and time $(t)$. A suspension levitator requires sensors, controllers, coils and the passage of current through a circuit structured specifically for an object to be suspended at a certain distance and weight. In Fig. 1, the design that gives us an idea of the forces that occur is shown.

The magnetic force equation that is derived from [8]:

$\vec{f}_{m l}(\vec{l}, x)=\frac{i^{2}}{2} \frac{d L}{d x}$

Is assumed the dependence equation: $L=L_{1}+\frac{L_{0} x_{0}}{2}$ put this into the Equation (2) we obtain:

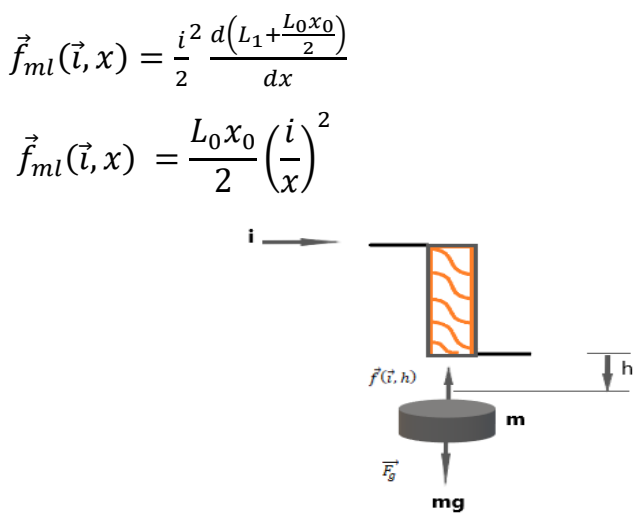

Fig. 1. Forces that are Applied in a Magnetic Levitation.
From the last equation we have the magnetic force constant $k=\frac{L_{0} x_{0}}{2}$

Being $i$ the current in the levitation coils, which control the force $\vec{f}(\vec{\imath}, x)$ and $x$ the gap-height or the distance of separation. Adding forces, following Newton's second law and considering the suspended object along $x$ axis, we have:

$\sum \vec{F}=m \vec{a}=m \ddot{y}=\vec{f}_{m l}(\vec{l}, x)-\overrightarrow{F_{g}}=0$

$m \ddot{y}=k\left(\frac{\vec{\imath}}{x}\right)^{2}-m g$

Where $i$ the current in the coil of the electromagnet is, $g$ is the gravitational constant, $k$ is the magnetic force constant and $m$ is the mass of the object.

The levitator consists of a magnet that create a magnetic field and electromagnets that control that magnetic field. It is necessary to vary the value of the electromagnetic force by adjusting the current that passes through the electromagnet because the electromagnet is responsible for generating the electromagnetic force that allows the levitation of the object, as it shown in Fig. 2.

Each magnet has two poles: the north and the south. Experiments show that opposites are attracted and the same poles repel each other. Four cylindrical magnets are placed in a square and have the same polarity, around forming a magnetic field up to push any magnet, which has the same pole and in the middle of them. Together with four levitation coils, placed equidistantly and symmetrical magnets, it is possible to create an opposite magnetic field.

Sets of dynamic equations (Newton's and Kirchhoff's equations) that provide theory to the system are given by the following set of equations using equation (4) we have the equation of mechanical system and the differential equation of the circuit.

$m \frac{d^{2} x}{d t^{2}}=m g-k\left(\frac{i}{x}\right)^{2}$

$L \frac{d i}{d t}=e(t)-R i$

Where $L$ is the inductance of the electromagnet that de, depends on the position of the object and a control variable, because is to assume that $L$ varies inversely with respect to the object's position $x$.

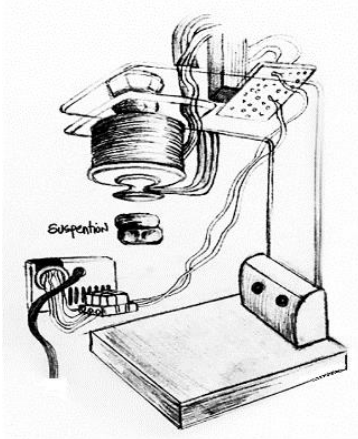

Fig. 2. Magnetic Suspension Levitator. 
The infrared photo-emitter and photo-receiver pair are the sensors used, which indicate that the amount of light received increases, which occurs when the metallic object moves away from the electromagnet, the output of the photo-receiver increases and vice versa. The equation that describes the sensor output $v_{S}$ is:

$v_{s}=k_{s} h+\bar{v}_{s}$

where $k_{s}>0$ indicates the gain of the sensor and $\overline{v_{S}}$ is constant and $h$ is the gap (Fig. 1).

The voltage applied to the electromagnet having the same linear form:

$i=k_{v} v+\bar{\imath}$

where $\bar{l}$ is the current for the sensor output to be $\bar{v}_{s}$.

The controller (photodiode) uses the sensor signal to adjust the voltage applied to the actuator and thus maintain levitation. For MISO systems or for each dynamic systems, where the knowledge of the enter states variables determine the behavior of the system in equation (1) see the set of state equations, linearizing we have:

$\dot{x_{1}}(t)=A(t) x(t)+B(t) e(t)$

$\dot{x_{2}}(t)=C(t) x(t)+D(t) e(t)$

Constructed the state equation we use the follow system:

$x_{1}=h, \quad x_{2}=\frac{d h}{d x}, \quad x_{3}=i$

We re write equation (5-6):

$\dot{x}=x_{1}, \quad \dot{x}_{1}=g-\frac{k}{m}\left(\frac{x_{2}}{x_{1}}\right)^{2}, \quad \dot{x}_{2}=\frac{e}{L}-\frac{R}{L} x_{2}$

We find the linearized state equations at the equilibrium point for temporary invariant systems, in the state space, where the variables $\tilde{x} y \tilde{e}$ are the variables subtracting the equilibrium positions.

$\widetilde{\mathbb{X}}=\mathbb{A} \widetilde{\mathbb{X}}+\mathbb{B} \widetilde{\mathbb{e}}, \mathbb{A}=\left[\begin{array}{cc}0 & 1 \\ \frac{2 g}{h_{0}} & 0\end{array}\right], \quad \mathbb{B}\left[\begin{array}{c}0 \\ \frac{-2 g \cdot k_{v}}{i_{0}}\end{array}\right]$

Where $\mathbb{A}$ and $\mathbb{B}$ are called matrix state.

\section{B. Electronic Circuit Design of the Magnetic Levitator}

For the design of the circuit, the following components shown in Table I was needed.

The Operational Amplifier is in the position of an inverter subtractor because in the circuit a high impedance is required to pass the current to stabilize it and maintain its constant flow, they are used more in devices with very weak signals, but where it requires a constant current flow. In addition, it requires 2 voltage sources, one in positive and one in negative for its operation, both with the same current.

$V_{\text {OUT }}=V_{2}\left(\frac{(R 3+R 1) R 4}{(R 4+R 2) R 1}\right)-V_{1}\left(\frac{R 3}{R 1}\right)$

Where:

- $\mathrm{R} 1=20 \mathrm{k} \Omega$

- $\mathrm{R} 2=20 \mathrm{k} \Omega$
- $\mathrm{R} 3=4.7 \mathrm{k} \Omega$

- $\mathrm{R} 4$ = Photodiode $($ Resistance converter based on the received light intensity), it is considered null.

- $\mathrm{V} 1=12 \mathrm{v} \& \mathrm{~V} 2=-12 \mathrm{~V}$

$V_{\text {OUT }}=2.079 \mathrm{~V}$

The output voltage of the Operational Amplifier is 2.079 $\mathrm{V}$, only and when the photodiode is activated, it will be supplied to the TRIAC to send it to the coil.

The TRIAC or Triode [9], is a switch capable of switching alternating and continuous current, it is used when the control of the current flow in a circuit is required as shown in Fig. 3, and it also requires an activation voltage, being $2,079 \mathrm{~V}$ obtained from the previous analysis. In this case, it will be used as an actuator controlled by the photodiode and the LED, will identify the presence of an object and activate the current flow for the creation of the magnetic field.

The coil is where current will flow, it is also where a The main function of the coil is to keep the current stable until it discharges on its own, meaning that, if there is a sudden change in the current, the coil will dissipate the magnetic field for periods of time until finally extinguished. For this reason, a diode is connected in antiparallel with the coil, in this way and thanks to the coil inverting the direction of the current, it will circulate through the diode. Thanks to this flow, the inductor will be discharged in a controlled manner and will not appear over voltages. The circuit is shown in Fig. 4 using all the electronic devices.

Finally, to find the power in direct current the following formula will be used:

$P(t)=R \cdot I^{2}=\frac{V^{2}}{R}$

Which $\mathrm{R}$ is the equivalent resistance of the circuit and $\mathrm{I}$ is the controlled input current of the circuit.

TABLE I. ELECTRONIC DEVICES

\begin{tabular}{|c|c|}
\hline Resistors & $\begin{array}{ll}- & 1 \times 270 \Omega \\
\text { - } & 2 \times 20 \mathrm{k} \Omega \\
\text { - } & 1 \times 4.7 \mathrm{k} \Omega \\
\text { - } & 1 \times 5.6 \mathrm{k} \Omega \\
\text { - } & 1 \times 1 \mathrm{k} \Omega\end{array}$ \\
\hline Operational Amplifier & - $\quad$ LM358 \\
\hline Led Diode & - $\quad 2 \times$ Diode Led \\
\hline TRIAC o Triode & - IRF540 \\
\hline Electric Capacitor & $\begin{array}{ll}- & 1000 \mu \mathrm{f} \\
\text { - } & 4.7 \mu \mathrm{f}\end{array}$ \\
\hline Diodes & $\begin{array}{ll}\text { - } & \text { PHOTODIODE } \\
\text { - } & \text { 1N5401 }\end{array}$ \\
\hline Coil & - $\quad$ Coil $3 \mathrm{mH}$ \\
\hline Voltage Regulator & $\begin{array}{ll}\text { - } & 12 \text { volts y } 2 \text { amps } \\
\text { - } & -12 \text { volts y } 2 \text { amps }\end{array}$ \\
\hline
\end{tabular}




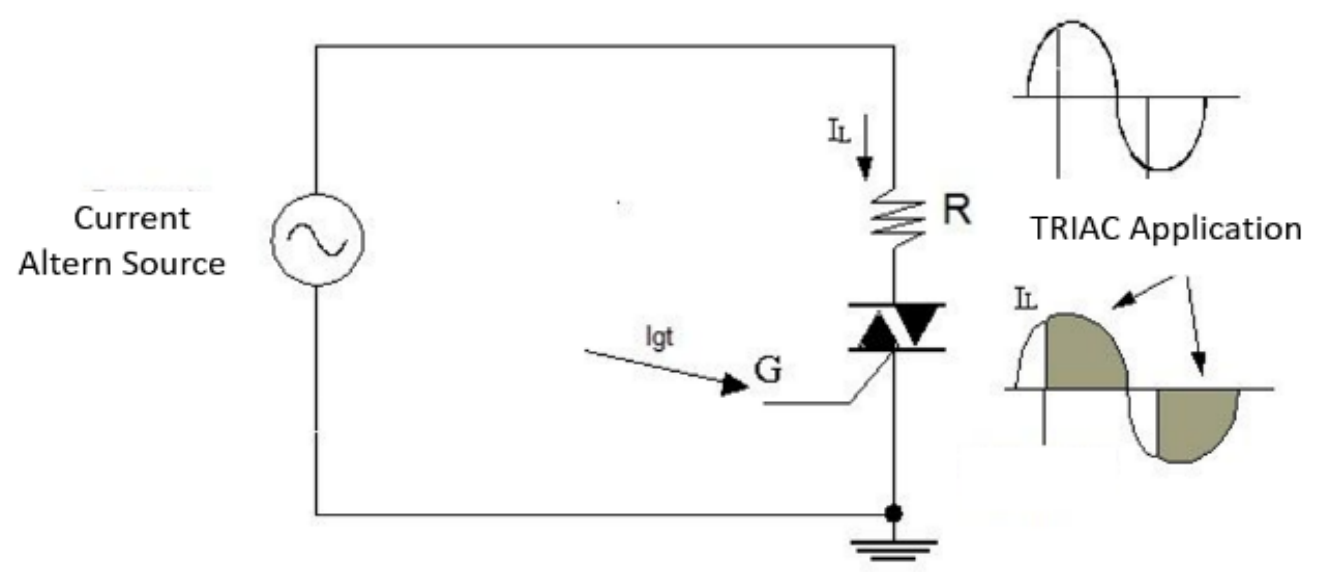

Fig. 3. Operation of the TRIAC Against the Current.

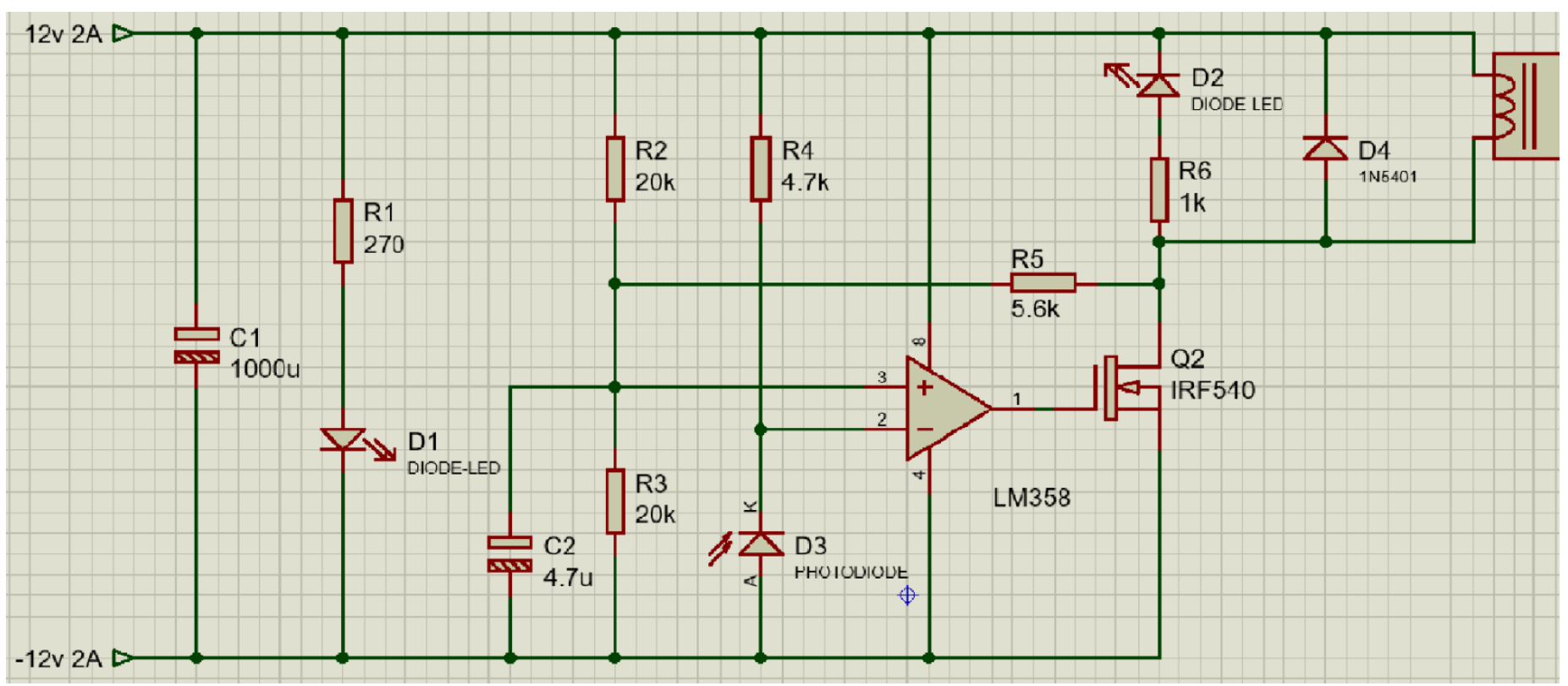

Fig. 4. Design of the Magnetic Levitator Circuit.

\section{RESULTS}

The electronic circuit of the magnetic levitator does not have loss of current due to the filters applied in the circuit to control the alternating current. In addition to establish the magnetic body within the range of the photodiode, providing stability and necessary use of current, the system is activated.

The simulation of the circuit was done through the software Proteus 8 , in which it does not provide connection or current distribution errors, besides controlling the high level of current with power circuits.

\section{DISCUSSION}

The research work confirms the use of electronic components without the need for a programmable microcontroller, the main purpose is to use electronic components based on their main function and identify the voltage and current values that run the circuit to know the final result for the power in the coil.

In electronic magnetic levitator circuit, it was considered the current level to get the optimum electromagnetic field in the coil, therefore, an OPAM (Operational Amplifier) was used to control the current and maintain the electric flow constant. In addition, the TRIAC is required front a very high current to preserve the level of current and take care of the other important electronic devices such as the coil. In case, it does not have these electronic devices implemented in the circuit, it cannot maintain the flow and stability of the current.

\section{CONCLUSIONS}

As a future work, we want to implement the electronic circuit and also add a magnetic levitator by repulsion to identify the sum of the forces exerted on an object when subjected by 2 electromagnetic fields. 
The use of the TRIAC is based on the level of operating current of the circuit, compared to a transistor because as a maximum it can work at 1 ampere, thus limiting the desired power in the coil to generate the electromagnetic field.

\section{ACKNOWLEDGMENTS}

We would like to thank Isabel Ivanka Cotrina she is the visual artist who drew the Fig. 2 in this document.

\section{REFERENCES}

[1] Nayak A., Controller design for magnetic levitation system, Thesis for Master, Department of Electrical Engineering NIT, India, April 2015.

[2] Giancoli, Douglas C.; Physics Principles with Applications, Person Education Limited, England, Chapter 16-21, 2016.

[3] M. Ahmed, M. F. Hossen, M. E. Hoque, O. Farrok, and M. Mynuddin; "Design and Construction of a Magnetic Levitation System Using Programmable Logic Controller," Am. J. Mech. Eng. Vol. 4, 2016, Pages 99-107, vol. 4, no. 3, pp. 99-107, May 2016.
[4] F. Grilli, A. Morandi, F. De Silvestri, and R. Brambilla; "Dynamic modeling of levitation of a superconducting bulk by coupled $\mathrm{H}$-magnetic field and Arbitrary Lagrangian-Eulerian formulations," Italy, 2018.

[5] J. A. Guijarro Solórzano and J. T. Vivar Martínez; "Diseño e Implementación de un Levitador Electro-Magnético basado en un Control PID utilizando LABVIEW," Universidad Politécnica Salesiana, Ecuador, 2015.

[6] S. Wojtczuk, X. Zhang, and W. MacNeish; "Enhanced visible nearinfrared photodiode and non-invasive physiological sensor," 2016.

[7] Castro-Beltrán J. S., Vergara Ramírez C. F, Herrera Guayazan J S; Comparison of a linear and nonlinear control on magnetic levitator; Ingenierías USBMed, vol. 9 (1), pag. 112-118, 2018.

[8] Silviu Folea, Cristina Muresan, Robin De Keyser, Clara Ionescu; Theoretical Analysis and Experimental Validation of a Simplified Fractional Order Controller for a Magnetic Levitation System, IEEE Transactions on control systems technology, 2015.

[9] S. Bárez-López, M. J. Obregón, R. Martínez de Mena, J. Bernal, A. Guadaño-Ferraz, and B. Morte; "Effect of TRIAC in the treatment of Mct8," MCT8 Symp., 2016. 